Dra. Esther Martínez Pastor

Universidad Rey Juan Carlos

@ Esther.martinez.pastor@urjc.es

(iD) $0000-0002-2861-750 \mathrm{X}$
- Recibido / Received 10 de octubre de 2018

- Aceptado / Acepted 6 de noviembre de 2018

- Páginas / Pages De la 163 a la 176

- ISSN: 1885-365X

\title{
Panorámica de las reclamaciones en publicidad y menores en Autocontrol (1998-2018)
}

A panoramic view of complaints in advertising and children in Autocontrol (2008-2018)

\begin{abstract}
Este artículo ofrece un panorama general de todas las reclamaciones presentadas ante la Asociación para la Autorregulación de la Comunicación Comercial (Autocontrol) sobre publicidad y menores durante los últimos veinte años (1998-2018). Se ha contado con una muestra de 68 casos. Mediante una metodología cuantitativa y cualitativa se han analizado las reclamaciones con relación a qué tipo de publicidad es la más reclamada ajustándose a la normativa de autorregulación, con qué argumentos se han fundamentado, qué medidas se han llevado a cabo por parte de Autocontrol y quiénes son los principales reclamantes y reclamados.
\end{abstract}

PALABRAS CLAVE: menores, normativa, autorregulación, publicidad.

This article offers an overview of all complaints filed with the Association for Self-Regulation of Commercial Communication (Autocontrol) on advertising and children during the last twenty years (1998-2018). There has been a sample of 68 cases. Through a quantitative and qualitative methodology the complaints have been analyzed in relation to the type of advertising complaint, the arguments on which they have based, what measures have been carried out by Autocontrol and who are the main complainant and complaint.

KEY WORDS: children, Advertising self-regulation (ASR), regulation, advertising.

\section{Introducción y estado de la cuestión}

En este artículo se analizan las reclamaciones sobre publicidad dirigida a los menores presentadas ante la Asociación para la Autorregulación de la Comunicación Comercial (Autocontrol) en las dos últimas décadas (1998-2018). El objetivo es conocer qué tipo de publicidad es la más reclamada ajustándose a la normativa de autorregulación, con qué argumentos se han fundamentado, qué medidas se han llevado a cabo por parte de Autocontrol y quiénes son los principales reclamantes y reclamados.

A su vez, se ofrece un panorama general sobre qué sectores incurren más la normativa 
de autorregulación en cuanto a la publicidad dirigida a menores. También, se recoge una radiografía diacrónica de las reclamaciones durante dos décadas en torno a este público objetivo.

Se ha utilizado la base de datos de Autocontrol para identificar las reclamaciones y se ha empleado como criterio de búsqueda las principales normativas de autorregulación relativas a los tipos de publicidad no permitidas que, a su vez, afecten a los menores.

Para llevar a cabo nuestro trabajo se han revisado los principales estudios en torno a este tema atendiendo a los siguientes criterios: trabajos relativos a autorregulación publicitaria en general, investigaciones focalizadas en la autorregulación publicitaria y menores, estudios centrados en la autorregulación publicitaria en menores y sectores concretos, y trabajos focalizados en los análisis de resoluciones de sistemas de autorregulación publicitaria como Autocontrol.

En cuanto a los estudios relativos a la autorregulación publicitaria en general, se destacan los estudios de Verbruggen (2012; 2011), Harker (1998), Burleton (1982), Harker y Harker (2002) y Boddewyn (1988; 1989; 1992) centrados en los organismos de autorregulación como autoridades de supervisión de la publicidad. En el ámbito nacional autores como Fernando Magarzo (2011) y Patiño Alves (2007) analizan la autorregulación desde una visión normativa y otras figuras afines. Otros investigadores como Feenestra y González Esteban (2017) estudian el funcionamiento del sistema de autorregulación español en términos de independencia, transparencia y participación. Martín Llaguno y Hernández Ruiz (2009) critican el exceso de autorregulación en nuestro país, mientras que otros trabajos comparan el sistema de autorregulación español con el de otros países como el norteamericano (Medina y An, 2012), el británico (Muela Molina y Perelló Oliver, 2014) y el portugués (Estrela y Loureiro, 2013) mostrando los puntos en común y las debilidades de los sistemas con una visión crítica y constructiva.

Lievens (2018) destaca la protección de los menores en el ámbito digital en los sistemas de autorregulación, corregulación y legislativo evidenciando la necesidad de identificar las responsabilidades de los diferentes actores en el ámbito publicitario. En esta misma línea, Macenaite (2016) analiza y reflexiona sobre las iniciativas de autorregulación adoptadas por la industria cuyo objeto es proteger la privacidad de los niños en la Red, evidenciando las fortalezas y debilidades del sistema. Otros autores como Verdoodt, Lambrecht y Lievens (2016) realizan una radiografía de las normas de autorregulación europeas en materia de menores mientras que Martínez Pastor, Vizcaíno, Serrano Maillo y Nicolás Ojeda (2017) realizan una panorámica normativa de la publicidad circunscrita al ámbito nacional.

También hay investigaciones en torno a la autorregulación en publicidad y a los menores vinculadas con determinados sectores como el de los alimentos o juguetes. Ejemplo de ello son los trabajos de Huizinga y Kruse (2016), Jensen y Ronit (2015), Hawkes y Harris (2011) que analizan el riesgo de la publicidad de alimentos no adecuada; o la investigación de Martínez Pastor, Gaona y Nicolás Ojeda (2017), Martínez Pastor y Nicolás Ojeda (2016) y Martínez Pastor, Nicolás Ojeda y Gaona (2014) centrada en la aplicación de la normativa de autorregulación de los juguetes en la publicidad televisiva de los juguetes.

Son menos copiosos los estudios relativos al análisis de las resoluciones de los sistemas de autorregulación publicitaria como Autocontrol. Entre los trabajos que han abordado estas líneas está el de Perelló Oliver y Muela Molina (2017) que recogen un análisis de las 
reclamaciones presentadas durante el período 2010-2015 en todos los sectores con el objeto de evidenciar los puntos débiles del sistema de autorregulación. En esta línea el estudio de Medina y An (2012) analiza las resoluciones de Autocontrol de los años 2005-2009 sin discriminar ningún sector. Otros autores se centran en las resoluciones vinculadas con la mujer como Pérez Marín (2011), y son escasos los trabajos que analizan las resoluciones de Autocontrol relativas a los menores entre los que encontramos los estudios de Martínez Pastor, Gaona y Nicolás Ojeda (2017) que analizan casos reclamados en publicidad y juguetes, o los trabajos de Pérez-Ugena y Coromina, Martínez Pastor y Perales Albert (2011) y Maroño Gargallo (2010) relativos a las resoluciones de los menores y al Código PAOS.

Estos trabajos evidencian y justifican el actual interés por conocer y analizar las resoluciones de Autocontrol relativas a los menores y seguir investigando en el tema de menores y autorregulación. Antes de entrar en el objeto de esta investigación conviene explicar cómo es el funcionamiento de Autocontroly las principales normas de autorregulación vinculadas directamente con los menores.

\section{Autocontrol: normativas y funcionamiento de las reclamaciones en torno a los menores}

La publicidad es una de las comunicaciones más regulada y autorregulada con el objeto de proteger al consumidor, tanto como público objetivo como potencial, por ser la parte más vulnerable, así como para promover una competencia leal en el mercado (De la Cuesta Rute, 2002) y fomentar una forma legal, honesta y responsable de la publicidad en el mercado (Tato Plaza, 2005; Fernando Magarzo, 2008). Las normas jurídicas que se centran en los límites en relación con la publicidad ilícita (art. 3, Ley 34/1988, de 11 de noviembre, General de Publicidad) se acotan en torno a los valores y derechos de la Constitución, el público objetivo -mujeres y menores-, el mensaje subliminal, engañoso, desleal y agresivo, y en el tipo de producto o servicio, mediante normas específicas. Los órganos de protección son el sistema jurídico y el sistema de autorregulación. Las normas jurídicas son vinculantes y obligadas para todos los afectados y todos los agentes que intervienen (Gómez Montero y Lema Devesa, 2010). Mientras que las normas de autorregulación se rigen principalmente por el Código de conducta publicitaria de Autocontrol (Código de Autocontrol, en adelante) y por los códigos sectoriales, así como por el carácter voluntario de cumplir estos códigos por parte de los que se adhieren a ellos.

Las empresas, anunciantes y agencias de publicidad de forma voluntaria y expresa se adhieren a la autorregulación, aprueban códigos de conducta éticos o deontológicos para el propio colectivo que se obligan a acatar y adoptan mecanismos adecuados para el control del cumplimiento de los códigos. Además, se sanciona a los miembros que no los cumplen. De este modo, se evita que la Administración regule o ejecute sentencias de un modo más rápido y duro que si la industria no se autorregulase.

Los principales códigos de autorregulación que protegen a los menores son: el Código de Autocontrol, que está basado en el Código internacional de prácticas publicitarias de la Cámara Internacional de Comercio (ICC,1996) y, los códigos que regulan los medios y a los 
sectores (Martínez Pastor, Vizcaíno Laorga, Serrano Maillo y Nicolás Ojeda, 2017). En el Código de Autocontrol las normas corresponden a los principios básicos que debe respetar todo mensaje publicitario, como el principio de legalidad, que significa que se debe respetar la ley y la Constitución, no abusar de la buena fe, no discriminar, no incitar a la violencia o no incitar a prácticas inseguras o peligrosas, entre otros; el principio de autenticidad, que exige que la publicidad debe ser identificable o; el principio de veracidad, que implica que la publicidad nunca debe ser engañosa, así como dispone de normas sobre determinadas formas y técnicas publicitarias, como la publicidad comparativa, agresiva, etc. (Martínez Pastor, 2010; Bel Mallén y Corredoira y Alfonso, 2003; Santaella López, 2003). De acuerdo con nuestro objeto de análisis, la norma de referencia del Códigos de Autocontrol es la norma 28:

La publicidad dirigida a niños deberá ser extremadamente cuidadosa. La misma no deberá explotar la ingenuidad, inmadurez, inexperiencia o credulidad natural de los niños o adolescentes, ni abusar de su sentido de la lealtad. La publicidad dirigida a los niños o adolescentes, o susceptible de influirles, no deberá contener declaraciones o presentaciones visuales que puedan producirles perjuicio mental, moral o físico. Se tendrá especial cuidado para asegurar que los anuncios no engañen o induzcan a error a los niños en lo que se refiere al tamaño real, valor, naturaleza, durabilidad y rendimiento del producto anunciado. Si se requiere el uso de otros artículos para su correcta utilización (por ejemplo, pilas) o para conseguir el resultado descrito o mostrado (por ejemplo, pintura) ello debe quedar explícitamente expresado. Los anuncios no deben sobrestimar el nivel de habilidad o el límite de edad de los niños para poder disfrutar o utilizar los productos.

Entre los códigos que regulan los medios se destacan el Código ético de confianza online que dedica su Título $\mathrm{V}$ a la «Protección de los menores» en cuanto a la forma de presentar los mensajes publicitarios, el contenido y la obtención de datos personales de los menores y, el Código de autorregulación sobre contenidos televisivos e infancia que trata la restricción horaria para contenidos y publicidad que no sea apropiada para la infancia y juventud.

Se destacan también los códigos sectoriales suscritos y promovidos por asociaciones de productos y servicios de determinados sectores y cuya aplicación se ha encomendado por la industria a Autocontrol como organismo independiente de autorregulación publicitaria: el Código de autorregulación de la publicidad infantil y juguetes (2015) y el Código de autorregulación de la publicidad de alimentos y bebidas dirigida a menores, prevención de la obesidad y salud (PAOS).

También hay otros códigos de autorregulación, que si bien no tienen como target al menor, sí consideran que éste puede ser destinatario de los mensajes publicitarios, por lo que se destaca en ellos el especial cuidado que la publicidad debe tener en caso de que pudiera ser vista por los menores, debido a su especial vulnerabilidad y credulidad. Se trata del Código de autorregulación del vino, el Código de autorregulación publicitaria de la Federación Española de Bebidas Espirituosas (FEBE) y el Código de autorregulación publicitaria de cerveceros de España. A estos también podemos sumarle, el Código de conducta sobre comunicaciones comerciales de las actividades de juego y las Directrices de buenas prácticas publicitarias de productos de software interactivo que protegen de forma especial a los menores como target al que se dirigen los productos anunciados. 


\section{Objetivos e hipótesis}

El objetivo principal del presente trabajo es conocer en que aspectos principales se fundamentan las reclamaciones de Autocontrol para estimar o desestimar los casos de publicidad y menores. Para ello es necesario conocer qué tipo de publicidad es la más reclamada en Autocontrol relativa a los menores, qué argumentos se fundamentan en las resoluciones, qué normativas de autorregulación se aplican y qué decisiones se han llevado a cabo por parte de los Jurados de Autocontrol.

Como objetivos secundarios se establece averiguar quiénes son los sujetos reclamantes y reclamados y los sectores más afectados de estas resoluciones.

La hipótesis de partida es que la mayoría de los casos reclamados sobre publicidad y menores no suele prosperar, ya sea porque el argumento deontológico del reclamante no se ajusta a la normativa, ya sea porque se realiza una interpretación laxa de la normativa. Además, la norma que se suele aplicar siempre es el Código de autorregulación de la publicidad en la norma 18 relativa a los menores y los códigos sectoriales relativos a juguetes.

\section{Metodología}

Se identifica la muestra de análisis que corresponde a las resoluciones tramitadas en Autocontrol y se analizan los datos obtenidos para dar respuesta al objetivo del presente trabajo.

El procedimiento utilizado para localizar los casos de publicidad y menores en el contexto español en los últimos veinte años (1998-2018) ha sido la base de datos de resoluciones del Jurado de Autocontrol. Se ha utilizado esta base de datos para la selección de casos de estudio ya que Autocontrol es el organismo donde se dirimen más casos de publicidad, generando una "jurisprudencia" mayor que la que se da en los tribunales ordinarios, por su rapidez y bajo coste frente a la justicia ordinaria. Para delimitar la búsqueda de los casos se han utilizado los siguientes criterios que están en la propia base de datos de Autocontrol referidos a la publicidad de productos y servicios en un lapso de tiempo concreto:

- Producto y/o servicio anunciado cuyo target es: infancia y juguetes, productos y servicios.

- Período y ámbito de la muestra: del 1 de enero de 1998 al 1 enero de 2018, España.

- Target de los anuncios: menores.

- Tipo de publicidad: se han seleccionado los ítems que están presentes en las normas de autorregulación de la publicidad y los menores siguiendo los estudios de Martínez Pastor, Vizcaíno Laorga, Serrano Maillo y Nicolás Ojeda (2017), Martínez Pastor, Gaona y Nicolás Ojeda (2017), Verdoodt, Lambrecht y Lievens (2016) y las normas de autorregulación anteriormente indicadas. A partir de estos criterios se identificaron estos tipos:

- Emplazamiento de producto.

- Encubierta. 
- Engañosa (en todas sus manifestaciones).

- Interpretación de frases tipo "hasta"...

- Infancia y menores de edad.

- Interpretación de anuncio y expresiones.

- Publicidad discriminatoria.

- Promoción publicitaria.

- Salud pública.

- Utilización de tipografías pequeñas.

En la recopilación de los casos se han tenido en cuenta tanto las resoluciones como los dictámenes del Jurado de Autocontrol. Conviene indicar que una de las características que definen estas resoluciones es que no siempre ambas partes, tanto el demandante como el demandado, forman parte de Autocontrol, de modo que los dictámenes que se dan, cuando una de las partes no forma parte, pueden libremente no aceptarse, mientras que en las resoluciones donde ambas partes son socios de Autocontrol sí deben acatarse. Asimismo, conviene indicar que para la selección de los casos no se han contabilizado aquellos que resultaban duplicados en la búsqueda cuando la Sección y el Pleno del Jurado han tenido la misma resolución. También es importante comprender que una vez que el Jurado de la sección tiene una resolución lo comunica a las partes y si una no está conforme puede solicitar un recurso de alza ante el Pleno del Jurado con otros miembros del Jurado. Otra cuestión a destacar es que de los casos localizados solo hemos tenido en cuenta los códigos de autorregulación que se aplican, ya que conviene recordar que el Jurado de Autocontrol únicamente tiene competencia para aplicar la normativa de autorregulación y nunca la ley porque su aplicación solo es posible en los tribunales ordinarios.

El universo lo configuran 1756 resoluciones y, aplicando los ítems anteriormente indicados, encontramos 73 casos. Una vez revisados los casos duplicados y descartados aquellos cuyos destinatarios de la publicidad reclamada no eran niños sino adultos la muestra final es de 68 casos resultados obtenidos de la base de datos de Autocontrol en materia de publicidad y menores.

La ficha de análisis se diseña a partir de las siguientes variables: caso en el que aparecen las partes implicadas (reclamante y reclamado), el sector, el motivo de la reclamación, los códigos deontológicos aplicados, los fundamentos deontológicos aplicados y, la resolución. Este análisis parte de un trabajo ya iniciado en el estudio de Martínez Pastor, Gaona y Nicolás Ojeda (2017) que analizaba solo los casos de publicidad y juguetes, mientras que en esta investigación la amplía a todos los sectores cuyo público objetivo sean menores durante dos décadas (1998-2018). 


\section{Resultados}

\subsection{Reclamantes}

Las partes reclamantes son de cuatro tipos: particulares, asociaciones, entidades públicas y empresas privadas.

Las asociaciones son los sujetos más activos ya que en los últimos veinte años han reclamado el $51,47 \%(n=35)$ de la totalidad, seguidos de las entidades públicas $30,88 \%$ ( $n=21)$ y con menor peso se sitúan los particulares que ha reclamado un $11,76 \%(n=8)$ y las empresas privadas $5,8 \%(n=4)$.

Las asociaciones que reclaman más una publicidad ajustada a la norma representan el $51,47 \%(n=35)$ del total de los reclamantes. Las más representativas son en primer lugar, la Comisión de Seguimiento de la Publicidad Infantil, con $51,4 \%(n=18)$ y configurada por Agencia Española de Consumo, Seguridad alimentaria y nutrición (AECOSAN), Comisión Nacional de los Mercados y la Competencia (CNMC), la Asociación Española de Anunciantes $(A E A)$, la Asociación de Usuarios de la Comunicación $(A U C)$ y la Asociación General de Consumidores (ASGECO) de acuerdo con el Código de autorregulación de la publicidad infantil de juguetes (CAPJ). Seguida de la Asociación de Usuarios de la Comunicación (AUC) con un $37,1 \%(n=13)$ del total de los casos reclamados por asociaciones. $Y$ en tercer lugar la Unión de Consumidores de la Comunitat Valenciana tiene una actividad relevante con 11,4 ( $n=4)$ de las reclamaciones interpuestas en los últimos veinte años. Esto responde a que el $C A P J$ crea una Comisión de seguimiento para evaluar la aplicación del Código y en caso de que se haya producido una infracción de las normas contenidas en él podrán presentar reclamaciones como así se constata en los casos identificados. En el caso de AUCy de la Unión de Consumidores de la Comunitat Valenciana son asociaciones cuyos objetivos son la defensa de los intereses de los ciudadanos y denunciar los casos de incumplimiento de las normas.

En el caso de las entidades públicas $(30,88 \%, n=21$ del total de la muestra) son los segundos que más reclaman ante Autocontrol por posibles infracciones. En este caso las entidades más activas son el Consell de L'Audiovisual de Catalunya (CAC) $(71,4 \%, \mathrm{n}=15)$, seguido de la Secretaría de Estado de Telecomunicaciones y para la Sociedad de la Información (SETSI) $(9,5 \%, \mathrm{n}=2)$, del Defensor del Menor de la Comunidad de Madrid (4,7\%, $\mathrm{n}=1)$ y otras $(14,2 \%, \mathrm{n}=3)$. Sin embargo, llama la atención la poca actividad en esta materia de los dos últimos entes públicos así como que el $C A C$ solo haya reclamado durante los años 1999 y 2000 y no haya más actividad por parte de este organismo en otros momentos.

Los particulares no son muy activos reclamando publicidad a pesar de los medios que hay a su disposición y que son gratuitos. La primera reclamación que se data es del año 2000 $(n=1)$ y posteriormente se encuentran tres casos reclamados durante 2016. Destaca la poca actividad de los ciudadanos aunque su ausencia puede deberse a que desconozcan que es un servicio gratuito para cualquier ciudadano accediendo desde la web de Autocontrol y que en la reclamación utilicen algún argumento deontológico o normativo para que pueda tramitarse.

Las empresas privadas solo reclaman en recurso de alzada contra la Sección que le ha instado a cesar o rectificar alguna pieza reclamada por una asociación, particular o empresa 
públicas. Este es el caso de El Corte Inglés o de Carrefour, que habiendo sido reclamados por la Unión de Consumidores de la Comunitat por publicidad discriminatoria en sus catálogos de Navidad y habiéndoles dado la razón el Jurado, realizaron un recurso de alzada contra la Sección del Jurado de Autocontrol sin ningún éxito.

\subsection{EL SECTOR}

Los sectores que se reclamaron durante el período analizado, según la clasificación de Infoadex, son: deportes y tiempo libre $(58,82 \%$; $n=40)$, distribución y restauración $(25 \%$; $n=17)$, alimentación $(4,40 \% ; n=3)$, cultura, enseñanza y medios de comunicación $(4,40 \%$; $n=3)$, hogar $(4,41 \% ; n=3)$, telecomunicación e internet y textil $(1,47 \% ; n=1)$ y vestimenta $(1,47 \% ; n=1)$. Estos sectores corresponden a productos o servicios como: restaurantes de niños (alimentación); películas (cultura, enseñanza y medios de comunicación); juguetes (deportes y tiempo libre); grandes almacenes (distribución y restauración); karaoke (hogar); aplicaciones (telecomunicación e internet y textil) y ropa (vestimenta).

Los juguetes, según el estudio de Martínez Pastor y Nicolás Ojeda (2016), son la categoría de producto más reclamado y en concreto los juegos de mesa $(30 \% ; n=12)$, seguido de muñecas y accesorios $(22,50 \% ; n=9)$, figuras de acción $(12,50 \% ; n=5)$ y construcciones $(10 \% ; n=4)$.

\subsection{El MOTIVO dE LA RECLAMACIÓN}

Los motivos de las reclamaciones son varios pero en la mayoría de los casos se alude a las normas del Código de conducta publicitaria de Autocontrol relativas al respeto del valor de la publicidad (norma 1), a la legalidad y a la Constitución (norma 2); a la interpretación de los anuncios (norma 3); a la buena fe (norma 4); a la publicidad discriminatoria (norma 10); a la publicidad engañosa (norma 14) y a la publicidad dirigida a niños y adolescentes (norma 28).

La forma más común es hacer alusión al principio de la legalidad (42,56\%; $n=29)$, norma 2 del Código de Autocontrol, porque en la gran mayoría se expresa la infracción de algún precepto y, por lo tanto, de la legalidad en términos generales. En segundo lugar, la norma más repetida en estas resoluciones son las relativas a la protección de los niños y adolescentes $(26,47 \%$; $n=18)$, norma 8 Código de Autocontrol, seguida de la publicidad engañosa $(19,11 \% ; n=13)$ por utilizar prescriptores o animación confusa que pueda generar engaño en los menores. Destacan $(13,23 \% ; n=9)$ los casos en los que se infringe el principio de publicidad discriminatoria, mientras que son menores los casos en los que se alude a los principios de buena fe $(11,76 \% ; n=8)$, interpretación de los anuncios $(2,94 \% ; n=1)$ y el valor de la publicidad $(2,94 \% ; n=1)$.

Además de estos motivos generales de las reclamaciones, en el caso de los juguetes son recurrentes los casos en los que se reclaman: la ficción animada en los anuncios que puede llevar a error a los menores en lo que respecta al juguete anunciado; el uso de prescriptores que abusan de la confianza que pueden tener estos sobre los menores; la utilización incorrecta del tamaño de los juguetes que puede no ser veraz a sus dimensiones, y el uso innecesario y exagerado de la violencia y de peligros que no son adecuados para los menores. 


\subsection{LoS CódIGOS DEONTOLÓGICOS APLICADOS Y NORMAS}

Los principales Códigos de autorregulación a los que se recurre en los casos de Autocontrol son: el Código de conducta publicitaria de Autocontrol (100\%; $\mathrm{n}=68)$ en todos los casos; el Código de autorregulación de la publicidad infantil de juguetes $(17,64 \% ; n=12)$ seguido del Código de autorregulación sobre contenidos televisivos e infancia $(2,41 \% ; n=2)$ y del Código ético de confianza online $(2,41 \% ; n=2)$. Llama la atención que el Código de autorregulación de la publicidad infantil de juguetes se utilice tan poco en las resoluciones de la muestra, así como la escasa utilización del Código ético de confianza online cuando actualmente hay numerosa publicidad en la Red y son casi inexistentes los casos en esta muestra.

\subsection{LA RESOLUCIÓN}

De los 68 casos, el 56\% ( $n=38)$ reclamaron ante la Sección del Jurado de Autocontrol y el $44 \%(n=30)$ interpusieron un recurso de alzada al Pleno. Del total de los casos el $50 \%$ $(n=34)$ fueron desestimados y el $50 \%(n=34)$ estimados. Llama la atención que de estas reclamaciones analizadas, el Pleno $(44 \% ; n=30)$ siempre esté de acuerdo con la Sección y no se haya encontrado ninguna resolución distinta. De los casos desestimados, el $41,1 \%$ $(n=28)$, Autocontrol les instó a la rectificación del anuncio $(60,71 \% ; n=17)$ o al cese del mismo $(39,28 \% ; n=11)$. Conviene indicar que durante el período seleccionado (2008-2018) las reclamaciones no responden a un incremento o descenso sino que su recorrido no es lineal y solamente hay un pico entre el año 1998 y 1999 sin entenderse el motivo.

Tabla 1. Resoluciones en materia de juguetes.

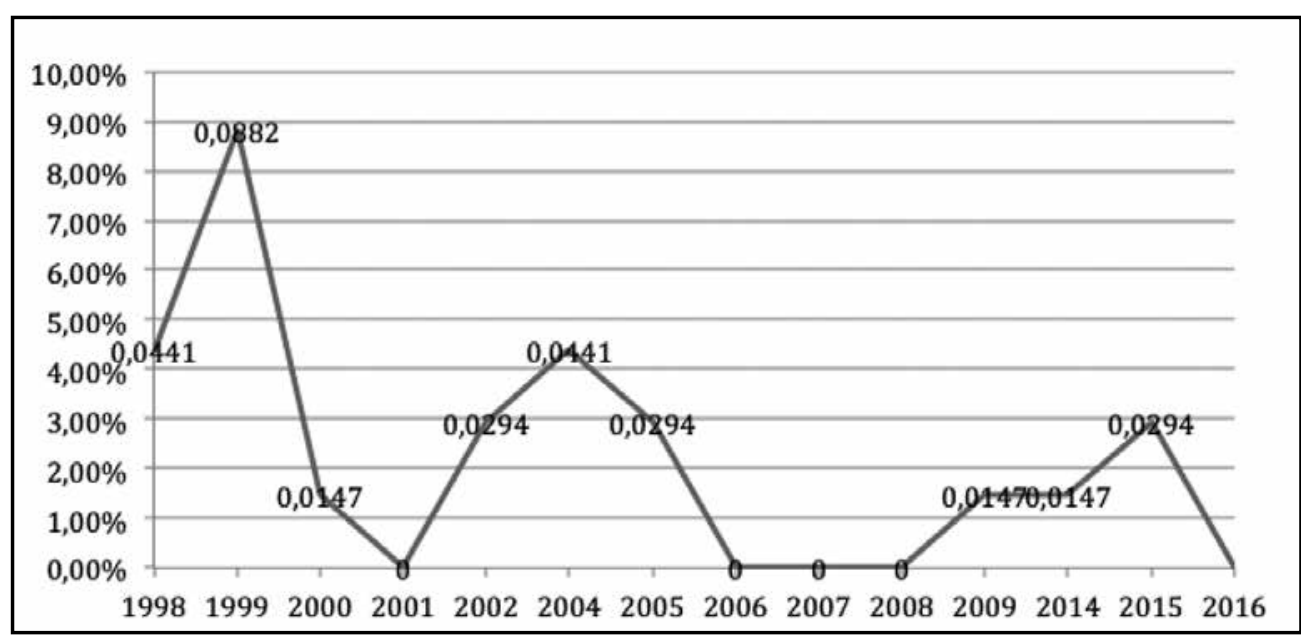

Elaboración propia a partir de las base de datos de Autocontrol 


\section{Discusión y conclusiones}

En primer lugar, debemos indicar que nuestra hipótesis de partida es nula dado que el $50 \%$ de las reclamaciones se estiman y el otro $50 \%$ de los casos el Jurado no cree que se infrinja ninguna norma deontológica. Sin embargo, los datos recogidos en el análisis ofrecen una descripción detallada que va más allá de reconocer si se reclama mucho o poco o si las resoluciones se estiman o no en una u otra medida. Dicha descripción parte del reconocimiento de las normas más utilizadas para establecer las resoluciones de los casos estudiados como son el Código de conducta publicitaria de Autocontrol, presente en todas las resoluciones, el Código de autorregulación de la publicidad infantil de juguetes, y el Código de autorregulación sobre contenidos televisivos e infancia, aunque este se aplica solo en dos casos en los que se emite publicidad en horario reforzado no adecuado para los menores.

El análisis nos ha permitido identificar que en el medio digital hay pocas resoluciones si se comparan con las resoluciones que afectan a medios como televisión o radio. Esto influye en la escasa utilización del Código ético de confianza para el medio digital, lo que resulta muy llamativo, ya que como destacan Martínez Pastor, Vizcaíno Laorga, Serrano Maillo y Nicolás Ojeda (2017), el incremento de la actividad publicitaria y sus modos en redes sociales condiciona nuevos escenarios y roles del menor ante la publicidad, no es solo espectador activo o pasivo sino que se ha convertido en un productor de contenidos comerciales.

Es importante destacar la participación de las asociaciones que defienden los intereses de los usuarios y consumidores y las entidades públicas, que son los sujetos que más reclaman ante Autocontrol, tal y como afirman Perelló Oliver y Muela Molina (2017) y Medina y An (2012). Por otra parte, resulta llamativa la escasa participación de los particulares ante Autocontrol, aunque en numerosas ocasiones son reclamaciones que no pueden estimarse porque no están fundamentadas en ninguna norma del Código de conducta publicitaria de Autocontrol o carecen de algún argumento deontológico.

También es llamativo el escaso volumen de reclamaciones de publicidad engañosa de juguetes en la última década frente a años anteriores. Consideramos que una explicación a este hecho pudiera estar en el incremento que se ha dado en los últimos años de los llamados Copy advice, lo que puede estar reduciendo el número de reclamaciones frente a décadas anteriores (Martínez Pastor, Gaona y Nicolás Ojeda, 2017). El servicio Copy advice de Autocontrol si bien es un control de consulta previa no vinculante es un requisito basado en Common Principles of Best Practice de la European Advertising Standards Alliance (EASA) (Autocontro). Se ha generado una práctica profesional por la cual se procura que el anunciante, bien a petición propia o de la agencia o del medio, compruebe antes de la emisión del anuncio si cumple o no la normativa. Por ejemplo, en 2015 se contabilizaron 1.947 Copy advice de spots de publicidad de juguetes y desde que se constituyó son 188.550 las consultas desarrolladas. Sin embargo, no debemos olvidar que este servicio es confidencial, voluntario y no vinculante, excepto en los códigos sectoriales que así lo prevean y, tan solo se emite un breve informe $u$ opinión de carácter confidencial que orienta al anunciante si el anuncio se ajusta a la norma. El Copy advice se suele solicitar en la etapa creativa por la empresa, la agencia o el medio. Generalmente es un script, storyboard o animatic de la misma a Autocontrol para evitar gastos si hubiera que hacer cambios. Sin embargo, un 
anuncio que ha pasado un Copy advice, y ha sido positivo, puede ser reclamado porque en la realización final de la pieza sea diferente al entregado al Copy advice y por lo tanto no se ajusta a lo que ideó en un principio.

En el ámbito de los juguetes, y con relación a las resoluciones, se encuentran temas recurrentes asociados en algunos casos con determinados productos, como son la utilización de animación confusa, el uso de prescriptores y la discriminación en los anuncios. Estos temas ya han sido abordados en estudios anteriores de Martínez Pastor, Gaona y Nicolás Ojeda (2017) y Martínez Pastor y Nicolás Ojeda (2016), donde se identifican potenciales infracciones de publicidad de juguetes emitidos en la televisión en diferentes períodos navideños. En cuanto a la animación, resulta llamativo que, si bien entendemos que las resoluciones de las Secciones y del Pleno se ajustan correctamente a las normativas de autorregulación, consideramos que debería replantearse una reflexión acerca del rol que desempeñan los destinatarios en este proceso. Creemos conveniente que el sector juguetero, la industria y Autocontrol debieran continuar en alerta frente a este tipo de prácticas. Es obligado preguntarse si realmente un menor es capaz de diferenciar de forma clara entre la ficción animada y la imagen real que hace referencia al producto o al menos de forma tan clara como pueda hacerlo un adulto. Por este motivo, es importante que se realice de forma muy clara la diferenciación para el menor. En estos casos la ficción animada no se asocia a un juguete en concreto, aunque en esta muestra se ha vinculado con muñecas y con figuras de acción. En relación con los prescriptores los fundamentos deontológicos siempre indican que un personaje famoso no debe instar a la compra de un juguete ni sugerir la compra del mismo por la inocencia del menor a creerse que es mejor porque lo anuncia un personaje famoso (Caso Karaoke Up Dance, 2004).

Por último, en relación con la discriminación de género la mayoría de los casos encontrados están asociados a los catálogos de navidad en los que las asociaciones reclaman a los grandes almacenes la diferenciación intencionada de géneros. En estas resoluciones el fundamento deontológico que se estimaba por discriminación se argumentaba de esta manera: «análisis del conjunto de un catálogo publicitario en el que la suma y unión global de la totalidad de las imágenes empleadas permitía apreciar una clara división y diferenciación dentro del mismo entre los bloques de juguetes que estarían destinados a niños y aquellos otros bloques que estarían destinados a niñas» (Caso Catálogo Navidades juguetes, 2015).

Tras los resultados obtenidos, consideramos de interés dirigir esta línea de trabajo hacia estudios comparados entre países que estudian la normativa europea y amplían así el conocimiento sobre el rol de los actores implicados, las normativas aplicadas, los argumentos y los sectores y productos sobre los que se aplican las resoluciones en un plano temporal más actual y menos diacrónico. 


\section{Bibliografía}

BEL MALLÉN, Ignacio y CORREDOIRA Y ALFONSO, Loreto (Coords.). (2003). Derecho de la información. Barcelona: Ariel.

BODDEWYN, Jean J. (1988). Advertising self-regulation and outside participation: A multinational comparison. New York: Quorum Book.

BODDEWYN, Jean J. (1989). Advertising self-regulation: True purpose and limits. Journal of Advertising, 18 (2), 19-27. doi: 10.1080/00913367.1989.10673148

BODDEWYN, Jean J. (1992). Global perspectives on advertising self-regulation. Principles and practices in thirty-eight countries. Connecticut: Quorum Books.

BURLETON, Eric (1982). The self-regulation of Advertising in Europe. Journal of Advertising, 1(4), 333-334.

DE LA CUESTA RUTE, José María (2002). Curso de derecho de la publicidad. Pamplona: Eunsa.

ESTRELA, Rui y LOUREIRO, Sandra M. C. (2013). Advertising self-regulation and complaints: A comparative study in Portugal and Spain. South Asian Journal of Marketing \& Management Research, 3(8), 68-79.

FEENSTRA, Ramón y GONZÁLEZ ESTEBAN, Elsa (2017). Autocontrol: A critical study of achievements and challenges in the pursuit of ethical advertising through an advertising self-regulation system. Journal of Business Ethics, 1-14. doi: 10.1007/s10551-016-3423-0

FERNANDO MAGARZO, María Rosario (2011). La consolidación de la autorregulación publicitaria en España: fomento normativo y reconocimiento juridisprudencial. Estudios sobre consumo, 84, 71-83.

GÓMEZ MONTERO, Jesús y LEMA DEVESA, Carlos (2010). Código de publicidad. Madrid: Marcial Pons.

HARKER, Debra (1998). Achieving acceptable advertising. International Marketing Review, 15(2), 101118. doi: $10.1108 / 02651339810212476$

HARKER, Debra y HARKER, Michael (2002). Dealing with complaints about advertising in Australia: The importance of regulatory self-discipline. International Journal of Advertising, 21(1), 23-45. doi: 10.1080/02650487.2002.11104915

HAWKES, Corinna y HARRIS, Jennifer (2011). An analysis of food industry pledges on marketing to children. Public Healths Nutrition, 14(8):1403-1414. doi: 10.1017/S1368980011000607

HUIZINGA, Oliver y KRUSE, Michaela (2016). Food industry self-regulation scheme "EU Pledge" cannot prevent the marketing of unhealthy foods to children. Obesity Medicine, 1: 24-28. doi: 10.1016/j. obmed.2016.01.004 
JENSEN, Jorgen D. y RONIT, Karsten (2015). The EU pledge for responsible marketing of food and beverages to children: implementation in food companies. European Journal of Clinical Nutrition, 69 (8), 896-901. doi: 10.1038/ejcn.2015.52

LIEVENS, Eva (2018). Research for CULT Committee - Solutions and policy dilemmas regardingminors' protection online. European Parliament, Policy Department for Structural and Cohesion Policies: Brussels.

MACENAITE, Milda (2016). Protecting children's privacy online: a critical look to four European self-regulatory initiatives. European Journal of Law and Technology, 7(2). Recuperado de: http://ejlt.org/article/view/473

MARTíN LLAGUNO, Marta y HERNÁNDEZ RUIZ, Alejandra (2009). El control de la comunicación comercial en un mundo globalizado: Regulación, autorregulación e hiperregulación de la publicidad. Portal de la Comunicación. Recuperado de: http://hdl.handle.net/10045/25698

MARTÍNEZ PASTOR, Esther (2010). Los mensajes publicitarios analizados desde la comunicación y el derecho. Madrid: Universitas.

MARTÍNEZ PASTOR, Esther, GAONA, Carmen y NICOLÁS OJEDA, Miguel Ángel (2017). Gestión pública y privada de la publicidad de juguetes. Regulación y autorregulación en la Unión Europea y España. Gestión y política pública, 26(2), 453-490. Recuperado de: https://dialnet.unirioja.es/servlet/articulo?codigo=6236814

MARTÍNEZ PASTOR, Esther y NICOLÁS OJEDA, Miguel Ángel (2016). Publicidad y juguetes: Un análisis de la aplicación de los códigos deontológicos y jurídicos en los spots de juguetes durante la Navidad 2015-2016. Madrid: OMM Campus Libros.

MARTÍNEZ PASTOR, Esther, NICOLÁS OJEDA, Miguel Ángel y GAONA, Carmen (2014). Informe de la publicidad de juguetes en televisión dirigidas a los menores durante las navidades 2013-2014: Desde el punto de vista deontológico y jurídico. Recuperado en http://www.aefj.es/noticias/?id=394

MARTÍNEZ PASTOR, Esther, VIZCAÍNO LAORGA, Ricardo, SERRANO MAILLO, Isabel y NICOLÁS OJEDA, Miguel Ángel (2017). Los niños ante la publicidad. Madrid: OMM Press.

MAROÑO GARGALLO, María del Mar (2010). El ámbito de aplicación del "Código de autorregulación de la publicidad de alimentos dirigidos a menores, prevención de la obesidad y salud" (Código PAOS). En: GÓMEZ SEGADE, José Antonio y GARCÍA VIDAL, Ángel. El derecho mercantil en el umbral del siglo XXI. Libro homenaje al Prof. Dr. Carlos Fernández-Novoa con motivo de su octogésimo cumpleaños. Madrid: Marcial Pons, pp. 365-378.

MEDINA, Mercedes y AN, Soontae (2012). Advertising self-regulation activity: A Comparision between Spain and US. ZER, 17(33), 13-29. Recuperado de: http://dadun.unav.edu/handle/10171/27403

MUELA MOLINA, Clara y PERELLÓ OLIVER, Salvador (2014). Advertising self-regulation. A comparative 
analysis between the United Kingdom and Spain. Communication \& Society, 27(3), 1-18. Recuperado de: https://www.unav.es/fcom/communication-society/es/resumen.php?art_id=498

PATIÑO ALVES, Beatriz (2007). La autorregulación publicitaria: especial referencia al sistema. Barcelona: Bosch.

PERELLÓ OLIVER, Salvador y MUELA MOLINA, Clara (2017). Advertising self-regulation (ASR) in Spain. An analysis of complaints and resolutions. Journal of Marketing Communications, 1-17. doi: 10.1080/13527266.2017.1337041

PÉREZ MARÍN, Inés (2011). Autorregulación: análisis de las resoluciones del jurado de autocontrol de la publicidad discriminatoria de la mujer. En: LÓPEZ DE LA CRUZ, Laura y OTERO CRESPO, Marta (Coords.), GARCíA RUBIO, María Paz y VALPUESTA FERNÁNDEZ, María del Rosario (Dirs.). El levantamiento del velo: las mujeres en el derecho privado. Valencia: Tirant lo Blanch, pp. 983-1016.

PÉREZ-UGENA Y COROMINA, Álvaro, MARTÍNEZ PASTOR, Esther y PERALES ALBERT, Alejandro (2011). Análisis y propuestas a partir del caso PAOS. Telos, Julio - Septiembre, 1-13. Recuperado de: https:/telos.fundaciontelefonica.com/archivo/numero088/analisis-y-propuestas-a-partir-del-caso-paos

SANTAELLA LÓPEZ, Manuel (2003). Derecho de la publicidad. Madrid: Civitas.

TATO PLAZA, Anxo (2005). La autodisciplina publicitaria. Revista Autocontrol de la Publicidad, 102.

VERBRUGGEN, Paul (2011). Transnational private regulation in the advertising industry. Case study conducted within the framework of the Research Project Constitutional Foundations of Transnational Private Regulation. Recuperado de: https://goo.gl/iAjKjW

VERBRUGGEN, Paul (2012). Enforcement of transnational private regulation of advertising practices: Decentralizations, mechanisms and procedural fairness. En: CAFAGGI, Fabrizio (Ed.). Enforcement of transnational regulation: Ensuring compliance in a global world. Edward Elgar Publishing: Cheltenham, pp. 302-327.

VERDOODT, Valerie, LAMBRECHT, Ingrid y LIEVENS, Eva (2016). Mapping and analysis of the current self and co-regulatory framework of commercial communication aimed at minors. $A$ report in the framework of the AdLit research project. Recuperado de http://www.AdLit.be 